\title{
Existence and uniqueness of solutions for systems of fractional differential equations with Riemann-Stieltjes integral boundary condition
}

\author{
Xinqiu Zhang ${ }^{1}$, Lishan $\mathrm{Liu}^{1,2^{*}}$, Yonghong $\mathrm{Wu}^{2}$ and Yumei Zou ${ }^{3}$
}

*Correspondence:

mathlls@163.com

'School of Mathematical Sciences,

Qufu Normal University, Qufu,

People's Republic of China

${ }^{2}$ Department of Mathematics and

Statistics, Curtin University, Perth,

Australia

Full list of author information is

available at the end of the article

\begin{abstract}
In this article, we first establish an existence and uniqueness result for a class of systems of nonlinear operator equations under more general conditions by means of the cone theory and monotone iterative technique. Furthermore, the iterative sequence of the solution and the error estimation of the system are given. Then we use this new result to study the existence and uniqueness of the solution for boundary value problems of systems of fractional differential equations with a Riemann-Stieltjes integral boundary condition in real Banach spaces. The results obtained in this paper are more general than many previous results and complement them.

MSC: 47H07; 47H10; 47J25; 34A08; 34B18; 34G20

Keywords: Systems of nonlinear binary operator equations; Real Banach spaces; Monotone iterative technique; Existence and uniqueness of positive solutions; Systems of fractional differential equations
\end{abstract}

\section{Introduction}

In this paper, we first study the following system of nonlinear operator equations in a real Banach space $E$ by means of the cone theory and monotone iterative technique:

$$
\left\{\begin{array}{l}
x=A(x, x), \\
x=B(x, x),
\end{array}\right.
$$

where $A, B: D \times D \rightarrow E$ are two nonlinear operators, $D$ is a subset of $E$. There have appeared a series of research results concerning the nonlinear operator equation $x=A(x, x)$ and $x=A x$ (see, for example, [37]) or the sum of several classes of mixed-monotone operator equations.

In [37], by using the cone theory and Banach contraction mapping principle, Zhang investigated the existence and uniqueness of solutions for a class of nonlinear operator equations $x=A x$ in real Banach spaces. The result is obtained only in the case that the

(c) The Author(s) 2018. This article is distributed under the terms of the Creative Commons Attribution 4.0 International License (http://creativecommons.org/licenses/by/4.0/), which permits unrestricted use, distribution, and reproduction in any medium, provided you give appropriate credit to the original author(s) and the source, provide a link to the Creative Commons license, and indicate if changes were made. 
cone $P$ is generating and normal and the operator A satisfies

$$
-B^{n_{0}}(x-y) \leq A x-A y \leq B^{n_{0}}(x-y), \quad \forall x, y \in E, x \geq y,
$$

where $A: E \rightarrow E$ is a nonlinear operator, $B: E \rightarrow E$ is a positive linear bounded operator, $n_{0}$ is a positive integer.

However, the upper-lower solutions conditions play an important role in [37]. Instead of supposing the upper-lower solutions conditions, they used a generating normal cone, which strengthened the conditions. Thus, how to remove these conditions is an interesting and important question. In this paper, compared with [37], we get Lemma 3.2 for which we shall not suppose the upper-lower solutions conditions, the generating of the cone and compactness of the operators. Here, by means of the cone theory and the Banach contraction mapping principle, the existence of a unique solution of the system of nonlinear operator binary equations (1.1) is investigated, furthermore, the iterative sequence of the solution and the error estimation of the system are given. The theorems obtained in this paper are more general than many previous results and complement them.

Fractional differential equations, arising in the mathematical modeling of systems and processes, have drawn more and more attention of the research community due to their numerous applications in various fields of science such as engineering, chemistry, physics, mechanics, etc. Boundary value problems of fractional differential equations have been investigated for many years (see $[9,17,20,27,29,36])$. Now, there are many papers dealing with the problem for different kinds of boundary value conditions such as multi-point boundary condition (see $[7,8]$ ), integral boundary condition (see [14-16, 19, 22, 25, 26, $30]$ ), and many other boundary conditions (see [12, 31, 32]). In recent years, the existence and uniqueness theorems of solutions for boundary value problems of nonlinear fractional differential equations have been studied extensively in the literature, mainly by using the fixed point theorem of the mixed-monotone operator (see, for instance, [7, 18, 33, 34] and their references), a priori estimate method and a maximal principle (see, for instance, [2]), the Banach contraction mapping principle and the Krasnose'skii fixed point theorem (see, for instance, $[1,10,11,17])$.

Here, we use the new result obtained in this paper to study the existence and uniqueness theorems of solutions for systems of the following simple fractional differential equations with a Riemann-Stieltjes integral boundary condition in real Banach space $E$ :

$$
\left\{\begin{array}{l}
-D_{0+}^{\alpha} u(t)=f_{1}\left(t, u(t), v(t), D_{0+}^{\beta_{i}} u(t), D_{0+}^{\gamma_{i}} v(t)\right), \quad 0<t<1, n-1<\alpha \leq n, \\
-D_{0+}^{\alpha} v(t)=f_{2}\left(t, v(t), u(t), D_{0+}^{\beta_{i}} v(t), D_{0+}^{\gamma_{i}} u(t)\right), \\
u^{(\kappa)}(0)=0, \quad D_{0+}^{\beta} u(1)=\int_{0}^{1} k(s) D_{0+}^{\beta_{n-1}} u(s) d A(s), \\
v^{(\kappa)}(0)=0, \quad D_{0+}^{\beta} v(1)=\int_{0}^{1} k(s) D_{0+}^{\beta_{+}} v(s) d A(s), \quad \kappa=0,1,2, \ldots, n-2,
\end{array}\right.
$$

where $n \geq 2, D_{0+}^{\alpha}, D_{0+}^{\beta_{i}}, D_{0+}^{\gamma_{i}}, D_{0+}^{\beta}, D_{0+}^{\gamma}$ are the standard Riemann-Liouville derivatives. $i-$ $1<\beta_{i}, \gamma_{i} \leq i(i=1,2, \ldots, n-1), \alpha-\beta_{n-1}>\alpha-\beta>1 . k:(0,1) \rightarrow \mathbb{R}_{+}$is continuous with $k \in L^{1}(0,1)$, and $\int_{0}^{1} k(s) u(s) d A(s)$ is the Riemann-Stieltjes integral of $u$ with respect to a function $A$ of bounded variation. In the following, we denote $I=[0,1] . f_{i}: I \times E^{4} \rightarrow E$ $(i=1,2)$ (we do not assume the continuity of $f_{i}$ ), for all $u, v \in C[I, E], f_{i}(\cdot, u(\cdot), v(\cdot)): I \rightarrow E$ is continuous. 
By means of monotone iterative technique and cone theory, we obtain some new existence theorems of the solutions and iterative approximation of the unique solution for the system of fractional differential equations with a Riemann-Stieltjes integral boundary condition, which does not possess any upper and lower solutions conditions in ordered Banach spaces. From this paper, we can see that the fixed point theorems in this paper have extensive applied background.

\section{Preliminaries}

Now we present briefly some definitions and basic results that are to be used in the article for convenience of the reader. We refer the reader to [3-6] for more details.

Suppose that $(E,\|\cdot\|)$ is a real Banach space, $\theta$ is the zero element of $E$. Recall that a non-empty closed convex set $P \subset E$ is a cone if it satisfies (1) $x \in P, \lambda \geq 0 \Rightarrow \lambda x \in P$; (2) $x \in P,-x \in P \Rightarrow x=\theta$. The real Banach space $E$ can be partially ordered by a cone $P \subset E$, i.e., $x \leq y$ if and only if $y-x \in P$. If $x \leq y$ and $x \neq y$, then we denote $x<y$ or $y>x$. Let $C[I, E]=\{x(t): I \rightarrow E \mid x(t)$ is continuous $\}$. Then $C[I, E]$ is a Banach space with the norm $\|x\|_{c}=\max _{t \in I}\|x(t)\|$, for $x \in C[I, E]$.

Moreover, $P$ is called normal if there exists a constant $N>0$ such that, for all $x, y \in E$, $\theta \leq x \leq y$ implies $\|x\| \leq N\|y\|$, the smallest $N$ is called the normality constant of $P$. If $x_{1}, x_{2} \in E$ with $x_{1} \leq x_{2}$ the set $\left[x_{1}, x_{2}\right]=\left\{x \in E \mid x_{1} \leq x \leq x_{2}\right\}$ is called the order interval between $x_{1}$ and $x_{2}$.

Definition 2.1 $([4,6])$ Let $D$ be a subset of a real Banach space $E . A: D \times D \rightarrow E$ is said to be a mixed-monotone operator if $A(x, y)$ is increasing in $x$, and decreasing in $y$, i.e., for all $x_{i}, y_{i} \in P(i=1,2)$ with $x_{1} \leq x_{2}, y_{1} \geq y_{2}$ imply $A\left(x_{1}, y_{1}\right) \leq A\left(x_{2}, y_{2}\right)$. The element $x \in D$ is called a fixed point of $A$ if $A(x, x)=x$.

\section{Lemmas}

Lemma 3.1 Let $E$ be a real Banach space, $P$ be a normal cone in $E, D=\left[u_{0}, v_{0}\right]=\{x \in E \mid$ $\left.u_{0} \leq x \leq v_{0}\right\}$ be the order interval in E. Assume that $A, B: D \times D \rightarrow E$ are two operators and satisfy the following conditions:

$\left(\mathrm{H}_{0}\right) u_{0} \leq A\left(u_{0}, v_{0}\right), B\left(v_{0}, u_{0}\right) \leq v_{0}$.

$\left(\mathrm{H}_{1}\right)$ For fixed $x \in D, A(x, y)$ and $B(x, y)$ are decreasing in $y$, and there exist two positive numbers $M_{i}>0(i=1,2)$ such that, for fixed $y \in D$, and for any $x_{1}, x_{2} \in D$ with $x_{1} \leq$ $x_{2}$,

$$
\begin{aligned}
& A\left(x_{2}, y\right)-A\left(x_{1}, y\right) \geq-M_{1}\left(x_{2}-x_{1}\right) \\
& B\left(x_{2}, y\right)-B\left(x_{1}, y\right) \geq-M_{2}\left(x_{2}-x_{1}\right) .
\end{aligned}
$$

$\left(\mathrm{H}_{2}\right)$ There exist a positive number $M_{3}>0$ and a positive integer $n_{0}$ such that, for all $x, y \in D$ with $x \leq y$,

$$
-M_{3}(y-x) \leq B(y, x)-A(x, y) \leq L^{n_{0}}(y-x)
$$

where $L: E \rightarrow E$ is a positive bounded linear operator with $r(L)<1$. 
Then the system of nonlinear operator equations (1.1) have a unique solution $\left(x^{*}, x^{*}\right)$ in $D \times D$. And for any initial values $x_{0}, y_{0} \in D$ with $x_{0} \leq y_{0}$, by constructing successively the sequences as follows:

$$
\left\{\begin{array}{l}
x_{n}=\frac{1}{1+M}\left[A\left(x_{n-1}, y_{n-1}\right)+M x_{n-1}\right], \\
y_{n}=\frac{1}{1+M}\left[B\left(y_{n-1}, x_{n-1}\right)+M y_{n-1}\right], \quad n=1,2, \ldots,
\end{array}\right.
$$

where $M=\max \left\{M_{1}, M_{2}, M_{3}\right\}>0$, we have $x_{n} \rightarrow x^{*}, y_{n} \rightarrow x^{*}$ in $E$, as $n \rightarrow \infty$. Moreover, for any $\delta: r(L)<\delta<1$, there exists a positive integer $n_{1}$ such that

$$
\begin{cases}\left\|x_{n}-x^{*}\right\| \leq 2 N\left(\frac{\delta^{n_{0}}+M}{1+M}\right)^{n}\left\|v_{0}-u_{0}\right\|, & n \geq n_{1}, \\ \left\|y_{n}-x^{*}\right\| \leq 2 N\left(\frac{\delta^{n} 0+M}{1+M}\right)^{n}\left\|v_{0}-u_{0}\right\|, & n \geq n_{1} .\end{cases}
$$

Proof Taking $M=\max \left\{M_{1}, M_{2}, M_{3}\right\}$. Let

$$
\left\{\begin{array}{l}
F(x, y)=\frac{1}{1+M}[A(x, y)+M x], \\
G(y, x)=\frac{1}{1+M}[B(y, x)+M y], \quad x, y \in D,
\end{array}\right.
$$

then (3.2) can be written as

$$
\left\{\begin{array}{l}
x_{n}=F\left(x_{n-1}, y_{n-1}\right), \\
y_{n}=G\left(y_{n-1}, x_{n-1}\right) .
\end{array}\right.
$$

By $\left(\mathrm{H}_{1}\right)$, we can easily prove that $F$ and $G$ satisfy the following conditions:

$\left(\mathrm{A}_{1}\right) F, G: D \times D \rightarrow E$ are two mixed-monotone operators.

$\left(\mathrm{A}_{2}\right)$ For all $x, y \in D$ with $x \leq y$, we have

$$
\begin{aligned}
& G(y, x)-F(x, y) \\
& \quad=\frac{1}{1+M}[B(y, x)+M y]-\frac{1}{1+M}[A(x, y)+M x] \\
& \quad=\frac{1}{1+M}[(B(y, x)-A(x, y))+M(y-x)] .
\end{aligned}
$$

Combining with $\left(\mathrm{H}_{2}\right)$, we can easily prove that

$$
\theta \leq G(y, x)-F(x, y) \leq H(y-x), \quad \forall x, y \in D, x \leq y,
$$

where $H \triangleq \frac{1}{1+M}\left(L^{n_{0}}+M I\right)$ in which $I$ is the identity operator.

$\left(\mathrm{A}_{3}\right)$ By $\left(\mathrm{H}_{0}\right)$, we have

$$
\begin{aligned}
& F\left(u_{0}, v_{0}\right)=\frac{1}{1+M}\left[A\left(u_{0}, v_{0}\right)+M u_{0}\right] \geq \frac{1}{1+M}\left[u_{0}+M u_{0}\right]=u_{0}, \\
& G\left(v_{0}, u_{0}\right)=\frac{1}{1+M}\left[B\left(v_{0}, u_{0}\right)+M v_{0}\right] \leq \frac{1}{1+M}\left[v_{0}+M v_{0}\right]=v_{0},
\end{aligned}
$$

thus, combining with (3.7), we have

$$
u_{0} \leq F\left(u_{0}, v_{0}\right) \leq G\left(v_{0}, u_{0}\right) \leq v_{0}
$$


Let $u_{n}=F\left(u_{n-1}, v_{n-1}\right), v_{n}=G\left(v_{n-1}, u_{n-1}\right)(n=1,2, \ldots)$. Thus, by $(3.10)$, we know

$$
u_{0} \leq u_{1} \leq v_{1} \leq v_{0}
$$

Therefore, by $\left(A_{1}\right)$ and $\left(A_{2}\right)$, using the method of the introduction, we can easily prove that

$$
u_{0} \leq u_{1} \leq \cdots \leq u_{n} \leq \cdots \leq v_{n} \leq \cdots v_{1} \leq v_{0}
$$

The proof will be divided into four steps.

Step 1: We prove that

$$
\theta \leq v_{n}-u_{n} \leq H^{n}\left(v_{0}-u_{0}\right), \quad n=1,2, \ldots
$$

Firstly, by $\left(A_{2}\right)$, we can easily prove that

$$
\theta \leq v_{1}-u_{1} \leq G\left(v_{0}, u_{0}\right)-F\left(u_{0}, v_{0}\right) \leq H\left(v_{0}-u_{0}\right),
$$

i.e., (3.13) hold for $n=1$. Suppose that (3.13) hold for $n=k$, i.e.,

$$
\theta \leq v_{k}-u_{k} \leq H^{k}\left(v_{0}-u_{0}\right)
$$

Then, for $n=k+1$, by $\left(\mathrm{A}_{1}\right)$ and $\left(\mathrm{A}_{2}\right)$ we know

$$
\begin{aligned}
& u_{k+1}=F\left(u_{k}, v_{k}\right) \leq G\left(v_{k}, u_{k}\right)=v_{k+1} \\
& \theta \leq v_{k+1}-u_{k+1}=G\left(v_{k}, u_{k}\right)-F\left(u_{k}, v_{k}\right) \leq H\left(v_{k}-u_{k}\right) \leq H^{k+1}\left(v_{0}-u_{0}\right) .
\end{aligned}
$$

By (3.14)-(3.16) and the method of the introduction, we know that (3.13) holds.

Step 2: We prove that $\left\{u_{n}\right\}$ is a Cauchy sequence. In view of $r(L)<1$, we know there exists a positive constant $\delta$ which satisfies $r(L)<\delta<1$. Thus

$$
\lim _{n \rightarrow \infty}\left\|H^{n}\right\|^{\frac{1}{n}}=r(H) \leq \frac{1}{1+M}\left(r\left(L^{n_{0}}\right)+M\right)<\frac{\delta^{n_{0}}+M}{1+M} \triangleq \delta_{0}<1,
$$

therefore, there exists a positive integer $n_{1}$ such that

$$
\left\|H^{n}\right\|<\delta_{0}^{n}, \quad n \geq n_{1}
$$

Then, by (3.12), we have

$$
\theta \leq u_{n} \leq u_{n+p} \leq v_{n+p} \leq v_{n}, \quad n, p=1,2, \ldots
$$

Consequently, by (3.13) and (3.18), we have

$$
\begin{aligned}
& \theta \leq u_{n+p}-u_{n} \leq v_{n}-u_{n} \leq H^{n}\left(v_{0}-u_{0}\right), \\
& \theta \leq v_{n}-v_{n+p} \leq v_{n}-u_{n} \leq H^{n}\left(v_{0}-u_{0}\right), \quad n, p=1,2, \ldots
\end{aligned}
$$


Therefore, by the normality of cone $P$ and (3.19), we have

$$
\begin{aligned}
& \left\|u_{n+p}-u_{n}\right\| \leq N\left\|H^{n}\left(v_{0}-u_{0}\right)\right\| \leq N \delta_{0}^{n}\left\|v_{0}-u_{0}\right\|, \\
& \left\|v_{n}-v_{n+p}\right\| \leq N\left\|H^{n}\left(v_{0}-u_{0}\right)\right\| \leq N \delta_{0}^{n}\left\|v_{0}-u_{0}\right\|, \quad n \geq n_{1}, p=1,2, \ldots,
\end{aligned}
$$

where $N$ is the normality constant of $P$. Consequently, $\left\{u_{n}\right\}$ and $\left\{v_{n}\right\}$ are two Cauchy sequences. Since $E$ is complete, there exist $u^{*}, v^{*} \in E$ such that

$$
\lim _{n \rightarrow \infty} u_{n}=u^{*}, \quad \lim _{n \rightarrow \infty} v_{n}=v^{*} .
$$

And by (3.12), we know

$$
u_{n} \leq u^{*} \leq v^{*} \leq v_{n}, \quad n=0,1,2, \ldots
$$

thus, $u^{*}, v^{*} \in D$. By (3.19) and (3.22), we have

$$
\theta \leq v^{*}-u^{*} \leq v_{n}-u_{n} \leq H^{n}\left(v_{0}-u_{0}\right), \quad n=0,1,2, \ldots
$$

Thus, by the normality of cone $P$, we have

$$
\left\|v^{*}-u^{*}\right\| \leq N\left\|H^{n}\left(v_{0}-u_{0}\right)\right\| \leq N \delta_{0}^{n}\left\|v_{0}-u_{0}\right\| \rightarrow 0, \quad n \rightarrow \infty,
$$

and thus $u^{*}=v^{*}$. Let $x^{*}:=u^{*}=v^{*}$ and then, by $\left(\mathrm{A}_{1}\right)$ and $\left(\mathrm{A}_{2}\right)$, we have

$$
u_{n+1}=F\left(u_{n}, v_{n}\right) \leq F\left(x^{*}, x^{*}\right) \leq G\left(x^{*}, x^{*}\right) \leq G\left(v_{n}, u_{n}\right)=v_{n+1}, \quad n=1,2, \ldots
$$

Let $n \rightarrow \infty$ in the former inequality, we have $F\left(x^{*}, x^{*}\right)=G\left(x^{*}, x^{*}\right)=x^{*}$, therefore, by the definitions of $F$ and $G$, we have $x^{*}=A\left(x^{*}, x^{*}\right), x^{*}=B\left(x^{*}, x^{*}\right)$, i.e., $\left(x^{*}, x^{*}\right)$ is the solution of operator equation (1.1).

Step 3: We prove that $\left(x^{*}, x^{*}\right)$ is the unique solution of operator equations (1.1) in $D \times D$. In fact, suppose $(\bar{x}, \bar{x})$ is another solution of Eqs. (1.1) in $D \times D$, then, by $\left(\mathrm{A}_{1}\right)$ and the method of the introduction, we easily see that $u_{n} \leq \bar{x} \leq v_{n}(n=1,2, \ldots)$. Thus, by (3.21) and the normality of $P$, we have $\bar{x}=x^{*}$. Therefore, the operator equations (1.1) have a unique solution $\left(x^{*}, x^{*}\right)$ in $D \times D$.

Step 4: We prove that (3.3) holds. Since $x_{0}, y_{0} \in D$, i.e., $u_{0} \leq x_{0} \leq y_{0} \leq v_{0}$. Suppose

$$
u_{n-1} \leq x_{n-1} \leq y_{n-1} \leq v_{n-1}
$$

then, by $\left(\mathrm{A}_{1}\right)$ and the method of the introduction, we can easily prove that

$$
u_{n} \leq x_{n} \leq y_{n} \leq v_{n}, \quad n=0,1,2, \ldots
$$

By (3.13) and (3.25), we have

$$
\begin{aligned}
& \theta \leq x_{n}-u_{n} \leq v_{n}-u_{n} \leq H^{n}\left(v_{0}-u_{0}\right), \\
& \theta \leq x^{*}-u_{n} \leq v_{n}-u_{n} \leq H^{n}\left(v_{0}-u_{0}\right) .
\end{aligned}
$$


Thus, by (3.17), (3.26) and (3.27), we obtain

$$
\begin{aligned}
\left\|x_{n}-x^{*}\right\| & \leq\left\|x_{n}-u_{n}\right\|+\left\|x^{*}-u_{n}\right\| \\
& \leq 2 N\left\|H^{n}\left(v_{0}-u_{0}\right)\right\| \\
& \leq 2 N \delta_{0}^{n}\left\|v_{0}-u_{0}\right\| \\
& =2 N\left(\frac{\delta^{n_{0}}+M}{1+M}\right)^{n}\left\|v_{0}-u_{0}\right\|, \quad n \geq n_{1} .
\end{aligned}
$$

In the same way, we can prove that

$$
\left\|y_{n}-x^{*}\right\| \leq 2 N \delta_{0}^{n}\left\|v_{0}-u_{0}\right\|=2 N\left(\frac{\delta^{n_{0}}+M}{1+M}\right)^{n}\left\|v_{0}-u_{0}\right\|, \quad n \geq n_{1} .
$$

Consequently, by (3.28) and (3.29), we know that (3.3) holds. Therefore, the proof of Lemma 3.1 is completed.

Lemma 3.2 Let $E$ be a real Banach space, $P$ be a normal cone in $E$. Assume that $A, B$ : $P \times P \rightarrow P$ are two operators and satisfy the following conditions:

$\left(\mathrm{J}_{0}\right)$ For fixed $x \in P, A(x, y)$ and $B(x, y)$ are decreasing in $y$, and there exist two positive numbers $M_{i}>0(i=1,2)$ such that, for fixed $y \in P$, and for any $x_{1}, x_{2} \in P$ with $x_{1} \leq x_{2}$,

$$
\begin{aligned}
& A\left(x_{2}, y\right)-A\left(x_{1}, y\right) \geq-M_{1}\left(x_{2}-x_{1}\right) \\
& B\left(x_{2}, y\right)-B\left(x_{1}, y\right) \geq-M_{2}\left(x_{2}-x_{1}\right) .
\end{aligned}
$$

$\left(\mathrm{J}_{1}\right)$ There exist a positive bounded linear operator $L_{1}: E \rightarrow E$ with $r\left(L_{1}\right)<1$, a positive integer $m_{0}$ and $h \in P$ such that, for all $x \in P$,

$$
B(x, \theta) \leq L_{1}^{m_{0}} x+h
$$

$\left(\mathrm{J}_{2}\right)$ There exist a positive bounded linear operator $L_{2}: E \rightarrow E$ with $r\left(L_{2}\right)<1$, a positive integer $n_{0}$ and a positive number $M_{3}>0$ such that, for all $x, y \in P, x \leq y$,

$$
-M_{3}(y-x) \leq B(y, x)-A(x, y) \leq L_{2}^{n_{0}}(y-x)
$$

Then the system of nonlinear operator equations (1.1) have a unique positive solution $\left(x^{*}, x^{*}\right)$ in $\left[\theta, w_{0}\right] \times\left[\theta, w_{0}\right]$, where $w_{0}=\left(I-L_{1}^{m_{0}}\right)^{-1} h=\sum_{n=0}^{\infty} L_{1}^{m_{0} n} h$. And for any initial values $x_{0}, y_{0} \in\left[\theta, w_{0}\right], x_{0} \leq y_{0}$, by constructing successively the sequences as follows:

$$
\left\{\begin{array}{l}
x_{n}=\frac{1}{1+M}\left[A\left(x_{n-1}, y_{n-1}\right)+M x_{n-1}\right], \\
y_{n}=\frac{1}{1+M}\left[B\left(y_{n-1}, x_{n-1}\right)+M y_{n-1}\right], \quad n=1,2, \ldots
\end{array}\right.
$$

where $M=\max \left\{M_{1}, M_{2}, M_{3}\right\}>0$, we have $x_{n} \rightarrow x^{*}, y_{n} \rightarrow x^{*}$ in $E$, as $n \rightarrow \infty$. Moreover, for any $r\left(L_{2}\right)<\delta<1$, there exists a positive integer $n_{1}$ such that

$$
\begin{cases}\left\|x_{n}-x^{*}\right\| \leq 2 N\left(\frac{\delta^{n_{0}}+M}{1+M}\right)^{n}\left\|v_{0}-u_{0}\right\|, & n \geq n_{1} \\ \left\|y_{n}-x^{*}\right\| \leq 2 N\left(\frac{\delta^{n_{0}+M}}{1+M}\right)^{n}\left\|v_{0}-u_{0}\right\|, & n \geq n_{1}\end{cases}
$$


Proof From $\left(\mathrm{J}_{1}\right)$, we know $L_{1}(P) \subset P$ and $r\left(L_{1}\right)<1$, thus $r\left(L_{1}^{m_{0}}\right)<1$, therefore,

$$
w_{0}=\left(I-L_{1}^{m_{0}}\right)^{-1} h=\sum_{n=0}^{\infty} L_{1}^{m_{0} n} h \in P,
$$

consequently, $B\left(w_{0}, \theta\right) \leq L_{1}^{m_{0}} w_{0}+h=w_{0}$. On the other hand, since $A: P \times P \rightarrow P$, we have $A\left(\theta, w_{0}\right) \geq \theta$.

Taking $u_{0}=\theta, v_{0}=w_{0}$, then $A\left(\theta, v_{0}\right) \geq \theta$ and $B\left(v_{0}, \theta\right) \leq v_{0}$. Consequently, by Lemma 3.1, the conclusions hold. Therefore, the proof of Lemma 3.2 is completed.

\section{Main result}

Let $E$ be a real Banach space, $P$ be a normal cone in $E$. In this section, we consider the existence and uniqueness of the solution as well as iterative approximation of the system of fractional differential equations (1.3) with a Riemann-Stieltjes integral boundary condition in ordered Banach spaces $E$ :

Now we present briefly some definitions, lemmas, and basic results that are to be used in the article for convenience of the reader. We refer the reader to [13, 14, 21, 23-25, 28, 35] for more details.

Definition $4.1([13,21,23,24])$ The Riemann-Liouville fractional integral of order $\alpha>0$ of a function $u:(0,+\infty) \rightarrow \mathbb{R}$ is given by

$$
I_{0^{+}}^{\alpha} u(t)=\frac{1}{\Gamma(\alpha)} \int_{0}^{t}(t-s)^{\alpha-1} u(s) d s
$$

provided that the right-hand side is pointwise defined on $(0,+\infty)$.

Definition $4.2([13,21,23,24])$ The Riemann-Liouville fractional derivative of order $\alpha>$ 0 of a continuous function $u:(0,+\infty) \rightarrow \mathbb{R}$ is given by

$$
D_{0^{+}}^{\alpha} u(t)=\frac{1}{\Gamma(n-\alpha)}\left(\frac{d}{d t}\right)^{n} \int_{0}^{t} \frac{u(s)}{(t-s)^{\alpha-n+1}} d s,
$$

where $n=[\alpha]+1,[\alpha]$ denotes the integer part of the number $\alpha$, provided that the righthand side is pointwise defined on $(0,+\infty)$.

Lemma $4.3([13,21,23,24])$

(1) If $u \in L^{1}(0,1)$ and $\alpha>\beta>0$, then

$$
I_{0^{+}}^{\alpha} I_{0^{+}}^{\beta} u(t)=I_{0^{+}}^{\alpha+\beta} u(t), \quad D_{0^{+}}^{\beta} I_{0^{+}}^{\alpha} u(t)=I_{0^{+}}^{\alpha-\beta} u(t), \quad D_{0^{+}}^{\beta} I_{0^{+}}^{\beta} u(t)=u(t) .
$$

(2) If $u \in L^{1}(0,1)$ and $\alpha>\beta>0$, then $D_{0^{+}}^{\alpha} u(t)=0$ has the unique solution

$$
f(t)=c_{1} t^{\alpha-1}+c_{2} t^{\alpha-2}+\cdots+c_{n} t^{\alpha-n}
$$

where $c_{i} \in \mathbb{R}(i=0,1,2, \ldots, n), n=[\alpha]+1$. 
Lemma 4.4 ([13, 21, 23, 24]) Let $\alpha>0$ and let $f(x)$ be integrable. Then

$$
I_{0^{+}}^{\alpha} D_{0^{+}}^{\alpha} f(u)=f(u)+c_{1} u^{\alpha-1}+c_{2} u^{\alpha-2}+\cdots+c_{n} u^{\alpha-n},
$$

where $c_{i} \in \mathbb{R}(i=1,2, \ldots, n), n$ is the smallest integer greater than or equal to $\alpha$.

Lemma 4.5 ([19]) Let $f_{1}, f_{2}$ be as in (1.3), and $x(t)=D_{0^{+}}^{\beta_{n-1}} u(t), y(t)=D_{0^{+}}^{\beta_{n-1}} v(t)$. Then the problem (1.3) is transformed to the following equation:

$$
\left\{\begin{array}{l}
D_{0^{+}}^{\alpha-\beta_{n-1}} x(t)+f_{1}\left(t, I_{0+}^{\beta_{n-1}-n+2} x(t), I_{0+}^{\beta_{n-1}-n+2} y(t), I_{0+}^{\beta_{n-1}-\beta_{i}} x(t), I_{0+}^{\beta_{n-1}-\gamma_{i}} y(t)\right)=0, \\
D_{0^{+}}^{\alpha-\beta_{n-1}} y(t)+f_{2}\left(t, I_{0+}^{\beta_{n-1}-n+2} y(t), I_{0+}^{\beta_{n-1}-n+2} x(t), I_{0+}^{\beta_{n-1}-\beta_{i}} y(t), I_{0+}^{\beta_{n-1}-\gamma_{i}} x(t)\right)=0, \\
I_{0+}^{\beta_{n-1}-n+2} x(0)=0, \quad I_{0+}^{\beta-\beta_{n-1}-n+2} x(1)=\int_{0}^{1} l(s) x(s) d A(s), \\
I_{0+}^{\beta_{n-1}-n+2} y(0)=0, \quad I_{0+}^{\beta-\beta_{n-1}-n+2} y(1)=\int_{0}^{1} l(s) y(s) d A(s) .
\end{array}\right.
$$

Furthermore, assume that $0 \leq \delta<\frac{\Gamma\left(\alpha-\beta_{n-1}\right)}{\Gamma(\alpha-\beta)}$, then the solution of (4.4) is equivalent to the solution of the following fractional integral equation:

$$
\left\{\begin{array}{l}
x(t)=\int_{0}^{1} G(t, s) f_{1}\left(t, I_{0+}^{\beta_{n-1}-n+2} x(s), I_{0+}^{\beta_{n-1}-n+2} y(s), I_{0+}^{\beta_{n-1}-\beta_{i}} x(s), I_{0+}^{\beta_{n-1}-\gamma_{i}} y(s)\right) d s, \\
y(t)=\int_{0}^{1} G(t, s) f_{2}\left(t, I_{0+}^{\beta_{n-1}-n+2} y(s), I_{0+}^{\beta_{n-1}-n+2} x(s), I_{0+}^{\beta_{n-1}-\beta_{i}} y(s), I_{0+}^{\beta_{n-1}-\gamma_{i}} x(s)\right) d s,
\end{array}\right.
$$

where

$$
G(t, s)=K(t, s)+\frac{t^{\alpha-\beta_{n-1}-1}}{\frac{\Gamma\left(\alpha-\beta_{n-1}\right)}{\Gamma(\alpha-\beta)}-\delta} g_{a}(s),
$$

in which

$$
\begin{aligned}
& K(t, s)=\frac{1}{\Gamma\left(\alpha-\beta_{n-1}\right)} \begin{cases}t^{\alpha-\beta_{n-1}-1}(1-s)^{\alpha-\beta-1}, & 0 \leq t \leq s \leq 1, \\
t^{\alpha-\beta_{n-1}-1}(1-s)^{\alpha-\beta-1}-(t-s)^{\alpha-\beta_{n-1}-1}, & 0 \leq s \leq t \leq 1,\end{cases} \\
& \delta=\int_{0}^{1} t^{\alpha-\beta_{n-1}-1} k(t) d A(t), \\
& g_{a}(s)=\int_{0}^{1} K(t, s) k(t) d A(t) .
\end{aligned}
$$

Moreover, if $x(t)=D_{0^{+}}^{\beta_{n-1}} u(t), y(t)=D_{0^{+}}^{\beta_{n-1}} v(t)$ is the positive solution of (4.4) then $u(t)=$ $I_{0^{+}}^{\beta_{n-1}} x(t), v(t)=I_{0^{+}}^{\beta_{n-1}} y(t)$ is a positive solution of problem (1.3).

Lemma 4.6 ([19]) Let $0 \leq \delta<\frac{\Gamma\left(\alpha-\beta_{n-1}\right)}{\Gamma(\alpha-\beta)}$ and $g_{a}(s) \geq 0, s \in[0,1]$, the Green function $G(t, s)$ have the following properties:

(1) $G:[0,1] \times[0,1] \rightarrow \mathbb{R}_{+}$is continuous and $G(t, s)>0$ for all $t, s \in(0,1)$;

(2) For any $t, s \in[0,1]$, we have $t^{\alpha-\beta_{n-1}-1} \phi(s) \leq G(t, s) \leq \phi(s)$, where

$$
\phi(s)=K(1, s)+\frac{g_{a}(s)}{\frac{\Gamma\left(\alpha-\beta_{n-1}\right)}{\Gamma(\alpha-\beta)}-\delta}, \quad s \in[0,1] .
$$

In the following we need the following assumptions: 
$\left(\mathrm{H}_{1}\right) f_{i}: I \times E^{4} \rightarrow E$ is continuous and satisfies, for all $x_{i}, y_{i} \in E(i=1,2,3,4)$, with $y_{1} \geq x_{1}$, $y_{2} \leq x_{2}, y_{3} \geq x_{3}, y_{4} \leq x_{4}$,

$$
f_{i}\left(t, y_{1}, y_{2}, y_{3}, y_{4}\right)-f_{i}\left(t, x_{1}, x_{2}, x_{3}, x_{4}\right) \geq 0, \quad \forall t \in I, i=1,2
$$

$\left(\mathrm{H}_{2}\right)$ There exist three positive Lebesgue integrable functions $a, b, c \in L^{1}\left(I, \mathbb{R}_{+}\right)$such that for all $x, y \in E, t \in I$,

$$
f_{2}(t, x, \theta, y, \theta) \leq a(t) x+b(t) y+c(t) e,
$$

where $e$ is a unit element in $E$;

$\left(\mathrm{H}_{3}\right)$ There exist four constants $r_{i}>0(i=1,2,3,4)$ such that, for any $t \in I, x_{i}, y_{i} \in E(i=$ $1,2,3,4)$ with $x_{1} \leq y_{1}, x_{2} \geq y_{2}, x_{3} \leq y_{3}, x_{4} \geq y_{4}$,

$$
\begin{aligned}
0 & \leq f_{2}\left(t, y_{1}, y_{2}, y_{3}, y_{4}\right)-f_{1}\left(t, x_{1}, x_{2}, x_{3}, x_{4}\right) \\
& \leq r_{1}\left(y_{1}-x_{1}\right)+r_{2}\left(x_{2}-y_{2}\right)+r_{3}\left(y_{3}-x_{3}\right)+r_{4}\left(x_{4}-y_{4}\right) ;
\end{aligned}
$$

$\left(\mathrm{H}_{4}\right) \max _{t \in I} \int_{0}^{1}|\widetilde{G}(t, s)| d s<1, \max _{t \in I} \int_{0}^{1}|\bar{G}(t, \tau)| d \tau<1$, where

$$
\begin{aligned}
\widetilde{G}(t, s)= & \frac{1}{\Gamma\left(\beta_{n-1}-n+2\right)}\left(\int_{1}^{\tau} G(t, s) a(s)(s-\tau)^{\beta_{n-1}-n+1} d s\right) \\
& +\frac{1}{\Gamma\left(\beta_{n-1}-\beta_{i}\right)}\left(\int_{1}^{\tau} G(t, s) b(s)(s-\tau)^{\beta_{n-1}-\beta_{i}-1} d s\right), \\
\bar{G}(t, s)= & \frac{r_{1}+r_{2}}{\Gamma\left(\beta_{n-1}-n+2\right)}\left(\int_{1}^{\tau} G(t, s)(s-\tau)^{\beta_{n-1}-n+1} d s\right) \\
& +\frac{r_{3}+r_{4}}{\Gamma\left(\beta_{n-1}-\beta_{i}\right)}\left(\int_{1}^{\tau} G(t, s)(s-\tau)^{\beta_{n-1}-\beta_{i}-1} d s\right) .
\end{aligned}
$$

Theorem 4.7 Let E be a real Banach space, $P$ be a normal cone in E. Assume that the conditions $\left(\mathrm{H}_{1}\right)-\left(\mathrm{H}_{4}\right)$ are satisfied. Then the system of nonlinear differential equations (1.3) have the unique positive symmetry solution $\left(w^{*}, w^{*}\right) \in D \times D$, where $D=\left[\theta, w_{0}\right] \subset C[I, E]$, $w_{0}$ is defined as in Lemma 3.2. Moreover, for any initial functions $x_{0}, y_{0} \in D$, there exist monotone iteration sequences $\left\{x_{n}\right\}$ and $\left\{y_{n}\right\}$, such that $x_{n} \rightarrow w^{*}, y_{n} \rightarrow w^{*}$ in $C[I, E]$, as $n \rightarrow \infty$, where

$$
\left\{\begin{aligned}
x_{n}(t)= & \int_{0}^{1} G(t, s) f_{1}\left(t, I_{0+}^{\beta_{n-1}-n+2} x_{n-1}(s), I_{0+}^{\beta_{n-1}-n+2} y_{n-1}(s),\right. \\
& \left.I_{0+}^{\beta_{n-1}-\beta_{i}} x_{n-1}(s), I_{0+}^{\beta_{n-1}-\gamma_{i}} y_{n-1}(s)\right) d s, \\
y_{n}(t)= & \int_{0}^{1} G(t, s) f_{2}\left(t, I_{0+}^{\beta_{n-1}-n+2} y_{n-1}(s), I_{0+}^{\beta_{n-1}-n+2} x_{n-1}(s),\right. \\
& \left.I_{0+}^{\beta_{n-1}-\beta_{i}} y_{n-1}(s), I_{0+}^{\beta_{n-1}-\gamma_{i}} x_{n-1}(s)\right) d s, \\
& t \in I, n=1,2,3, \ldots
\end{aligned}\right.
$$

Proof It is well known that $(u, v) \in C[I, E] \times C[I, E]$ is a solution of the system (1.3) if and only if $(x, y) \in C[I, E] \times C[I, E]$ is a solution of the system of nonlinear integral equations

$$
\left\{\begin{array}{l}
x(t)=\int_{0}^{1} G(t, s) f_{1}\left(t, I_{0+}^{\beta_{n-1}-n+2} x(s), I_{0+}^{\beta_{n-1}-n+2} y(s), I_{0+}^{\beta_{n-1}-\beta_{i}} x(s), I_{0+}^{\beta_{n-1}-\gamma_{i}} y(s)\right) d s, \\
y(t)=\int_{0}^{1} G(t, s) f_{2}\left(t, I_{0+}^{\beta_{n-1}-n+2} y(s), I_{0+}^{\beta_{n-1}-n+2} x(s), I_{0+}^{\beta_{n-1}-\beta_{i}} y(s), I_{0+}^{\beta_{n-1}-\gamma_{i}} x(s)\right) d s .
\end{array}\right.
$$


Consider the operators $A, B: D \times D \rightarrow C[I, E]$ as follows:

$$
\begin{aligned}
A(x, y)(t)= & \int_{0}^{1} G(t, s) f_{1}\left(t, I_{0+}^{\beta_{n-1}-n+2} x(s), I_{0+}^{\beta_{n-1}-n+2} y(s),\right. \\
& \left.I_{0+}^{\beta_{n-1}-\beta_{i}} x(s), I_{0+}^{\beta_{n-1}-\gamma_{i}} y(s)\right) d s, \\
B(y, x)(t)= & \int_{0}^{1} G(t, s) f_{2}\left(t, I_{0+}^{\beta_{n-1}-n+2} y(s), I_{0+}^{\beta_{n-1}-n+2} x(s),\right. \\
& \left.I_{0+}^{\beta_{n-1}-\beta_{i}} y(s), I_{0+}^{\beta_{n-1}-\gamma_{i}} x(s)\right) d s, \\
& x, y \in D, t \in I .
\end{aligned}
$$

Then $D \times D \rightarrow C[I, E]$. By Lemma 4.6 and $\left(\mathrm{H}_{1}\right)$, for all $t \in I,\left(x_{1}, y_{1}\right),\left(x_{2}, u_{2}\right) \in D \times D$, $x_{1} \leq x_{2}, y_{1} \geq y_{2}$, we obtain

$$
\begin{aligned}
& A\left(x_{2}, y_{2}\right)(t)-A\left(x_{1}, y_{1}\right)(t) \\
& =\int_{0}^{1} G(t, s) f_{1}\left(t, I_{0+}^{\beta_{n-1}-n+2} x_{2}(s), I_{0+}^{\beta_{n-1}-n+2} y_{2}(s), I_{0+}^{\beta_{n-1}-\beta_{i}} x_{2}(s), I_{0+}^{\beta_{n-1}-\gamma_{i}} y_{2}(s)\right) d s \\
& \quad-\int_{0}^{1} G(t, s) f_{1}\left(t, I_{0+}^{\beta_{n-1}-n+2} x_{1}(s), I_{0+}^{\beta_{n-1}-n+2} y_{1}(s), I_{0+}^{\beta_{n-1}-\beta_{i}} x_{1}(s), I_{0+}^{\beta_{n-1}-\gamma_{i}} y_{1}(s)\right) d s \\
& \quad 00 \\
& B\left(x_{2}, y_{2}\right)(t)-B\left(x_{1}, y_{1}\right)(t) \\
& \quad=\int_{0}^{1} G(t, s) f_{2}\left(t, I_{0+}^{\beta_{n-1}-n+2} x_{2}(s), I_{0+}^{\beta_{n-1}-n+2} y_{2}(s), I_{0+}^{\beta_{n-1}-\beta_{i}} x_{2}(s), I_{0+}^{\beta_{n-1}-\gamma_{i}} y_{2}(s)\right) d s \\
& \quad-\int_{0}^{1} G(t, s) f_{2}\left(t, I_{0+}^{\beta_{n-1}-n+2} x_{1}(s), I_{0+}^{\beta_{n-1}-n+2} y_{1}(s), I_{0+}^{\beta_{n-1}-\beta_{i}} x_{1}(s), I_{0+}^{\beta_{n-1}-\gamma_{i}} y_{1}(s)\right) d s \\
& \geq 0 .
\end{aligned}
$$

Consequently, $A, B: D \times D \rightarrow C[I, E]$ are mixed-monotone. By $\left(\mathrm{H}_{2}\right)$, for all $x \in D, t \in I$, we obtain

$$
\begin{aligned}
B(x, \theta)(t)= & \int_{0}^{1} G(t, s) f_{2}\left(s, I_{0+}^{\beta_{n-1}-n+2} x(s), 0, I_{0+}^{\beta_{n-1}-\beta_{i}} x(s), 0\right) d s \\
\leq & \int_{0}^{1} G(t, s)\left(a(s) I_{0+}^{\beta_{n-1}-n+2} x(s)+b(s) I_{0+}^{\beta_{n-1}-\beta_{i}} x(s)+c(s) e\right) d s \\
\leq & \int_{0}^{1} G(t, s) a(s)\left(\frac{1}{\Gamma\left(\beta_{n-1}-n+2\right)} \int_{0}^{s}(s-\tau)^{\beta_{n-1}-n+1} x(\tau) d \tau\right) d s \\
& +\int_{0}^{1} G(t, s) b(s)\left(\frac{1}{\Gamma\left(\beta_{n-1}-\beta_{i}\right)} \int_{0}^{s}(s-\tau)^{\beta_{n-1}-\beta_{i}-1} x(\tau) d \tau\right) d s \\
& +e \int_{0}^{1} G(t, s) c(s) d s \\
= & \int_{0}^{1} \frac{1}{\Gamma\left(\beta_{n-1}-n+2\right)}\left(\int_{1}^{\tau} G(t, s) a(s)(s-\tau)^{\beta_{n-1}-n+1} d s\right) x(\tau) d \tau \\
& +\int_{0}^{1} \frac{1}{\Gamma\left(\beta_{n-1}-\beta_{i}\right)}\left(\int_{1}^{\tau} G(t, s) b(s)(s-\tau)^{\beta_{n-1}-\beta_{i}-1} d s\right) x(\tau) d \tau
\end{aligned}
$$




$$
\begin{aligned}
& +e \int_{0}^{1} G(t, s) c(s) d s \\
= & L_{1} x(t)+h(t),
\end{aligned}
$$

where

$$
\begin{aligned}
L_{1} x(t)= & \int_{0}^{1}\left[\frac{1}{\Gamma\left(\beta_{n-1}-n+2\right)}\left(\int_{1}^{\tau} G(t, s) a(s)(s-\tau)^{\beta_{n-1}-n+1} d s\right)\right. \\
& \left.+\frac{1}{\Gamma\left(\beta_{n-1}-\beta_{i}\right)}\left(\int_{1}^{\tau} G(t, s) b(s)(s-\tau)^{\beta_{n-1}-\beta_{i}-1} d s\right)\right] x(\tau) d \tau, \\
h(t)=e & \int_{0}^{1} G(t, s) c(s) d s .
\end{aligned}
$$

Set

$$
\begin{aligned}
\widetilde{G}(t, s)= & \frac{1}{\Gamma\left(\beta_{n-1}-n+2\right)}\left(\int_{1}^{\tau} G(t, s) a(s)(s-\tau)^{\beta_{n-1}-n+1} d s\right) \\
& +\frac{1}{\Gamma\left(\beta_{n-1}-\beta_{i}\right)}\left(\int_{1}^{\tau} G(t, s) b(s)(s-\tau)^{\beta_{n-1}-\beta_{i}-1} d s\right),
\end{aligned}
$$

then $L_{1} x(t)=\int_{0}^{1} \widetilde{G}(t, s) x(s) d s$. In the following we prove $r\left(L_{1}\right)<1$. In fact, by $\left(\mathrm{H}_{4}\right)$, since $\max _{t \in I} \int_{0}^{1}|\widetilde{G}(t, s)| d s<1$, there exists a constant $m_{1}: 0<m_{1}<1$ such that $\int_{0}^{1}|\widetilde{G}(t, s)| d s \leq$ $m_{1}<1$, for any $t \in I$. Thus, for all $t \in I, x \in D$,

$$
\begin{aligned}
\left\|\left(L_{1} x\right)(t)\right\| & =\left\|\int_{0}^{1} \widetilde{G}(t, s) x(s) d s\right\| \\
& \leq \int_{0}^{1}\|\widetilde{G}(t, s) x(s)\| d s \\
& \leq \int_{0}^{1}|\widetilde{G}(t, s)| d s\|x\|_{c} \\
& \leq m_{1}\|x\|_{c}, \quad t \in I, \\
\left\|\left(L_{1}^{2} x\right)(t)\right\| & =\left\|\int_{0}^{1} \widetilde{G}(t, s)\left(L_{1} x\right)(s) d s\right\| \\
& \leq \int_{0}^{1}\left\|\widetilde{G}(t, s)\left(L_{1} x\right)(s)\right\| d s \\
& \leq \int_{0}^{1}|\widetilde{G}(t, s)| d s\left\|\left(L_{1} x\right)(s)\right\| \\
& \leq\left(\int_{0}^{1}|\widetilde{G}(t, s)| d s\right) m_{1}\|x\|_{c} \\
& \leq m_{1}^{2}\|x\|_{c}, \quad t \in I .
\end{aligned}
$$

By the method of the introduction, we can easily prove that, for all natural numbers $n$,

$$
\left\|\left(L_{1}^{n} x\right)(t)\right\| \leq m_{1}^{n}\|x\|_{c}, \quad t \in I
$$


therefore

$$
\left\|L_{1}^{n} x\right\|_{c}=\max _{t \in I}\left\|\left(L_{1}^{n} x\right)(t)\right\| \leq m_{1}^{n}\|x\|_{c}
$$

consequently

$$
\left\|L_{1}^{n}\right\| \leq m_{1}^{n}
$$

thus, $r\left(L_{1}\right)=\lim _{n \rightarrow \infty}\left\|L_{1}^{n}\right\|^{\frac{1}{n}} \leq m_{1}<1$.

By $\left(\mathrm{H}_{3}\right)$, for any $t \in I, x, y \in D$ with $x \leq y$,

$$
\begin{aligned}
& B(y, x)(t)-A(x, y)(t) \\
& =\int_{0}^{1} G(t, s) f_{2}\left(t, I_{0+}^{\beta_{n-1}-n+2} y(s), I_{0+}^{\beta_{n-1}-n+2} x(s), I_{0+}^{\beta_{n-1}-\beta_{i}} y(s), I_{0+}^{\beta_{n-1}-\gamma_{i}} x(s)\right) d s \\
& \quad-\int_{0}^{1} G(t, s) f_{1}\left(t, I_{0+}^{\beta_{n-1}-n+2} x(s), I_{0+}^{\beta_{n-1}-n+2} y(s), I_{0+}^{\beta_{n-1}-\beta_{i}} x(s), I_{0+}^{\beta_{n-1}-\gamma_{i}} y(s)\right) d s \\
& \geq 0,
\end{aligned}
$$

$B(y, x)(t)-A(x, y)(t)$

$$
\begin{aligned}
= & \int_{0}^{1} G(t, s) f_{2}\left(t, I_{0+}^{\beta_{n-1}-n+2} y(s), I_{0+}^{\beta_{n-1}-n+2} x(s), I_{0+}^{\beta_{n-1}-\beta_{i}} y(s), I_{0+}^{\beta_{n-1}-\gamma_{i}} x(s)\right) d s \\
& -\int_{0}^{1} G(t, s) f_{1}\left(t, I_{0+}^{\beta_{n-1}-n+2} x(s), I_{0+}^{\beta_{n-1}-n+2} y(s), I_{0+}^{\beta_{n-1}-\beta_{i}} x(s), I_{0+}^{\beta_{n-1}-\gamma_{i}} y(s)\right) d s \\
\leq & \int_{0}^{1} G(t, s) r_{1}\left(I_{0+}^{\beta_{n-1}-n+2} y(s)-I_{0+}^{\beta_{n-1}-n+2} x(s)\right) d s \\
& +\int_{0}^{1} G(t, s) r_{2}\left(I_{0+}^{\beta_{n-1}-n+2} y(s)-I_{0+}^{\beta_{n-1}-n+2} x(s)\right) d s \\
& +\int_{0}^{1} G(t, s) r_{3}\left(I_{0+}^{\beta_{n-1}-\beta_{i}} y(s)-I_{0+}^{\beta_{n-1}-\beta_{i}} x(s)\right) d s \\
& +\int_{0}^{1} G(t, s) r_{4}\left(I_{0+}^{\beta_{n-1}-\beta_{i}} y(s)-I_{0+}^{\beta_{n-1}-\beta_{i}} x(s)\right) d s \\
= & \int_{0}^{1} G(t, s)\left(r_{1}+r_{2}\right) I_{0+}^{\beta_{n-1}-n+2}(y(s)-x(s)) d s \\
& +\int_{0}^{1} G(t, s)\left(r_{3}+r_{4}\right) I_{0+}^{\beta_{n-1}-\beta_{i}}(y(s)-x(s)) d s \\
= & \int_{0}^{1} G(t, s)\left(r_{1}+r_{2}\right)\left(\frac{1}{\Gamma\left(\beta_{n-1}-n+2\right)} \int_{0}^{s}(s-\tau)^{\beta_{n-1}-n+1}(y(\tau)-x(\tau)) d \tau\right) d s \\
& +\int_{0}^{1} G(t, s)\left(r_{3}+r_{4}\right)\left(\frac{1}{\Gamma\left(\beta_{n-1}-\beta_{i}\right)} \int_{0}^{s}(s-\tau)^{\beta_{n-1}-\beta_{i}-1}(y(\tau)-x(\tau)) d \tau\right) d s \\
= & \int_{0}^{1} \frac{r_{1}+r_{2}}{\Gamma\left(\beta_{n-1}-n+2\right)}\left(\int_{1}^{\tau} G(t, s)(s-\tau)^{\beta_{n-1}-n+1} d s\right)(y(\tau)-x(\tau)) d \tau \\
& +\int_{0}^{1} \frac{r_{3}+r_{4}}{\Gamma\left(\beta_{n-1}-\beta_{i}\right)}\left(\int_{1}^{\tau} G(t, s)(s-\tau)^{\beta_{n-1}-\beta_{i}-1} d s\right)(y(\tau)-x(\tau)) d \tau \\
& (t) \\
& \\
&
\end{aligned}
$$


where

$$
\begin{aligned}
L_{2} x(t)= & \int_{0}^{1}\left[\frac{r_{1}+r_{2}}{\Gamma\left(\beta_{n-1}-n+2\right)}\left(\int_{1}^{\tau} G(t, s)(s-\tau)^{\beta_{n-1}-n+1} d s\right)\right. \\
& \left.+\frac{r_{3}+r_{4}}{\Gamma\left(\beta_{n-1}-\beta_{i}\right)}\left(\int_{1}^{\tau} G(t, s)(s-\tau)^{\beta_{n-1}-\beta_{i}-1} d s\right)\right] x(\tau) d \tau .
\end{aligned}
$$

Set

$$
\begin{aligned}
\bar{G}(t, s)= & \frac{r_{1}+r_{2}}{\Gamma\left(\beta_{n-1}-n+2\right)}\left(\int_{1}^{\tau} G(t, s)(s-\tau)^{\beta_{n-1}-n+1} d s\right) \\
& +\frac{r_{3}+r_{4}}{\Gamma\left(\beta_{n-1}-\beta_{i}\right)}\left(\int_{1}^{\tau} G(t, s)(s-\tau)^{\beta_{n-1}-\beta_{i}-1} d s\right),
\end{aligned}
$$

then $L_{2} x(t)=\int_{0}^{1} \bar{G}(t, s) x(s) d s$. Consequently, for any $x, y \in D$ with $x \leq y$,

$$
\theta \leq B(y, x)-A(x, y) \leq L_{2}(y-x)
$$

Using the same method as in the proof of $r\left(L_{1}\right)<1$, we can prove that $r\left(L_{2}\right)<1$.

Thus all conditions of Lemma 3.2 are satisfied, therefore the conclusions of Theorem 4.7 hold. Consequently, the proof of Theorem 4.7 is completed.

Acknowledgements

The authors would like to thank the referees for their useful suggestions, which have significantly improved the paper.

Funding

The work are supported financially by the National Natural Science Foundation of China $(11371221,11571296)$.

Availability of data and materials

Data sharing not applicable to this article as no datasets were generated or analyzed during the current study.

Competing interests

The authors declare that they have no competing interests.

\section{Authors' contributions}

All authors contributed equally to each part of this work. All authors read and approved the final manuscript.

\section{Author details}

${ }^{1}$ School of Mathematical Sciences, Qufu Normal University, Qufu, People's Republic of China. ${ }^{2}$ Department of Mathematics and Statistics, Curtin University, Perth, Australia. ${ }^{3}$ Department of Statistics and Finance, Shandong University of Science and Technology, Qingdao, People's Republic of China.

\section{Publisher's Note}

Springer Nature remains neutral with regard to jurisdictional claims in published maps and institutional affiliations.

Received: 29 April 2018 Accepted: 30 May 2018 Published online: 08 June 2018

\section{References}

1. Cui, Y.J.: Uniqueness of solution for boundary value problems for fractional differential equations. Appl. Math. Lett. 51, 48-54 (2016)

2. Cui, Y.J., Zou, Y.M.: An existence and uniqueness theorem for a second order nonlinear system with coupled integral boundary value conditions. Appl. Math. Comput. 256, 438-444 (2015)

3. Deimling, K.: Nonlinear Functional Analysis. Springer, Berlin (1985)

4. Guo, D.J.: Fixed points of mixed monotone operators with application. Appl. Anal. 34, 215-224 (1988)

5. Guo, D.J., Lakshmikantham, V.: Nonlinear Problems in Abstract Cones. Academic Press, Boston (1988)

6. Guo, D.J., Lakskmikantham, V.: Coupled fixed points of nonlinear operators with applications. Nonlinear Anal. 11 , 623-632 (1987)

7. Guo, L.M., Liu, L.S., Wu, Y.H.: Existence of positive solutions for singular fractional differential equations with infinite-point boundary conditions. Nonlinear Anal., Model. Control 21(5), 635-650 (2016) 
8. Guo, L.M., Liu, L.S., Wu, Y.H.: Existence of positive solutions for singular higher-order fractional differential equations with infinite-points boundary conditions. Bound. Value Probl. 2016, 114 (2016)

9. Guo, L.M., Liu, L.S., Wu, Y.H.: Iterative unique positive solutions for singular $p$-Laplacian fractional differential equation system with several parameters. Nonlinear Anal., Model. Control 23(2), 182-203 (2018)

10. Henderson, J., Luca, R.: Existence of positive solutions for a singular fractional boundary value problem. Nonlinear Anal., Model. Control 22, 99-114 (2017)

11. Henderson, J., Luca, R.: Existence of nonnegative solutions for a fractional integro-differential equation. Results Math. $72,747-763(2017)$

12. Jiang, J.Q., Liu, L.S., Wu, Y.H.: Positive solutions to singular fractional differential system with coupled boundary conditions. Commun. Nonlinear Sci. Numer. Simul. 18(11), 3061-3074 (2013)

13. Kilbas, A.A., Srivastava, H.M., Trujillo, J.J.: Theory and Applications of Fractional Differential Equations, vol. 204. Elsevier, Amsterdam (2006)

14. Li, H.D., Liu, L.S., Wu, Y.H.: Positive solutions for singular nonlinear fractional differential equation with integral boundary conditions. Bound. Value Probl. 2015, 232 (2015)

15. Liu, L.S., Hao, X.Q., Wu, Y.H.: Positive solutions for singular second order differential equations with integral boundary conditions. Math. Comput. Model. 57(3-4), 836-847 (2013)

16. Liu, L.S., Li, H.D., Liu, C., Wu, Y.H.: Existence and uniqueness of positive solutions for singular fractional differential systems with coupled integral boundary value problems. J. Nonlinear Sci. Appl. 10, 243-262 (2017)

17. Liu, L.S., Sun, F.L., Zhang, X.G., Wu, Y.H.: Bifurcation analysis for a singular differential system with two parameters via to degree theory. Nonlinear Anal., Model. Control 22, 31-50 (2017)

18. Liu, L.S. Zhang, X. Jiang, J. Wu, Y.H.: The unique solution of a class of sum mixed monotone operator equations and its application to fractional boundary value problems. J. Nonlinear Sci. Appl. 9(5), 2943-2958 (2016)

19. Liu, X.Q., Liu, L.S., Wu, Y.H.: Existence of positive solutions for a singular nonlinear fractional differential equation with integral boundary conditions involving fractional derivatives. Bound. Value Probl. 2018, 24 (2018)

20. Mi, Y.Y., Huang, S.B., Huang, C.Y.: Combined effects of the Hardy potential and lower order terms in fractional Laplacian equations. Bound. Value Probl. 2018, 61 (2018)

21. Miller, K., Ross, B.: An Introduction to the Fractional Calculus and Fractional Differential Equations. Wiley, New York (1993)

22. Min, D.D., Liu, L.S., Wu, Y.H.: Uniqueness of positive solutions for the singular fractional differential equations involving integral boundary value conditions. Bound. Value Probl. 2018, 23 (2018)

23. Podlubny, I.: Fractional Differential Equations. Mathematics in Science and Engineering, vol. 198. Academic Press, New York (1999)

24. Samko, S., Kilbas, A., Marichev, O.: Fractional Integral and Derivative: Theory and Applications. Gordon \& Breach, Yverdon (1993)

25. Wang, Y., Liu, L.S., Zhang, X.G., Wu, Y.H.: Positive solutions of a fractional semipositone differential system arising from the study of HIV infection models. Appl. Math. Comput. 258, 312-324 (2015)

26. Wang, Y.Q., Liu, L.S., Wu, Y.H.: Positive solutions for a nonlocal fractional differential equation. Nonlinear Anal. 74(11), 3599-3605 (2011)

27. Wang, Y.Q., Liu, L.S., Wu, Y.H.: Positive solutions for a class of fractional boundary value problem with changing sign nonlinearity. Nonlinear Anal. 74(17), 6434-6441 (2011)

28. Yuan, C.J.: Multiple positive solutions for $(n-1, n)$-type semipositone conjugate boundary value problems of nonlinear fractional differential equations. Electron. J. Qual. Theory Differ. Equ. 2010, 36 (2010)

29. Zhang, X., Liu, L.S., Wu, Y.H.: Existence and uniqueness of iterative positive solutions for singular Hammerstein integral equations. J. Nonlinear Sci. Appl. 10, 3364-3380 (2017)

30. Zhang, X., Zhong, Q.Y.: Uniqueness of solution for higher-order fractional differential equations with conjugate type integral conditions. Fract. Calc. Appl. Anal. 20, 1471-1484 (2017)

31. Zhang, X.G., Liu, L.S., Wu, Y.H.: Multiple positive solutions of a singular fractional differential equation with negatively perturbed term. Math. Comput. Model. 55(3-4), 1263-1274 (2012)

32. Zhang, X.G., Liu, L.S., Wu, Y.H.: Existence results for multiple positive solutions of nonlinear higher order perturbed fractional differential equations with derivatives. Appl. Math. Comput. 219(4), 1420-1433 (2012)

33. Zhang, X.G., Liu, L.S., Wu, Y.H.: The uniqueness of positive solution for a singular fractional differential system involving derivatives. Commun. Nonlinear Sci. Numer. Simul. 18, 1400-1409 (2013)

34. Zhang, X.G., Liu, L.S., Wu, Y.H.: The uniqueness of positive solution for a fractional order model of turbulent flow in a porous medium. Appl. Math. Lett. 27, 26-33 (2014)

35. Zhang, X.G., Liu, L.S., Wu, Y.H., Lu, Y.N.: The iterative solutions of nonlinear fractional differential equations. Appl. Math. Comput. 219, 4680-4691 (2013)

36. Zhang, X.G., Liu, L.S., Wu, Y.H., Wiwatanapataphee, B.: The spectral analysis for a singular fractional differential equation with a signed measure. Appl. Math. Comput. 257, 252-263 (2015)

37. Zhang, X.Y.: Fixed point theorems for a class of nonlinear operators in real Banach spaces and applications. Nonlinear Anal. 69, 536-543 (2008) 